\title{
Cortisol Inhibits Neuroplasticity Induction in Human Motor Cortex
}

\author{
Martin V. Sale, Michael C. Ridding, and Michael A. Nordstrom \\ Discipline of Physiology and Research Centre for Human Movement Control, School of Molecular and Biomedical Science, The University of Adelaide, \\ Adelaide SA 5005, Australia
}

\begin{abstract}
We investigated whether plasticity of human motor cortex (M1) is influenced by time of day, and whether changes in circulating levels of cortisol contribute to this effect. Neuroplasticity was induced using paired associative stimulation (PAS), involving electrical stimulation of left median nerve, paired with transcranial magnetic stimulation over the right M1 25 ms later (90 pairs at 0.05 Hz). Surface EMG was recorded from the left abductor pollicis brevis (APB) and first dorsal interosseous muscle. Cortisol levels were assessed from saliva. Time-of-day modulation of PAS effectiveness was assessed in 25 subjects who were tested twice, at 8:00 A.M. and 8:00 P.M. on separate days. In a second double-blind study, 17 subjects were tested with PAS at 8:00 P.M. on two occasions after administration of oral hydrocortisone $(24 \mathrm{mg}$ ) or placebo. The motor-evoked potential (MEP) in resting APB increased significantly after PAS in the evening (when endogenous cortisol levels were low), but not in the morning. Oral hydrocortisone prevented facilitation of the APB MEP after PAS,

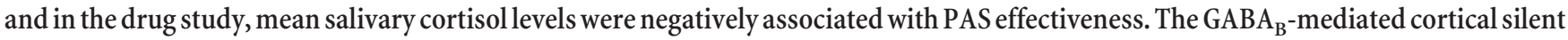
period for APB was longer in the morning than in the evening, and was lengthened by PAS and oral hydrocortisone. We conclude that neuroplasticity in human $\mathrm{M} 1$ and $\mathrm{GABA}_{\mathrm{B}}$-dependent intracortical inhibitory systems are influenced by time of day and modified by circulating levels of cortisol.
\end{abstract}

Key words: transcranial magnetic stimulation; cortisol; paired associative stimulation; circadian; plasticity; cortical inhibition

\section{Introduction}

Experimental techniques that induce neuroplastic changes in human motor cortex (M1) allow investigation of conditions thought to involve aberrant neuroplasticity, including focal dystonia (Quartarone et al., 2003) and Parkinson's disease (Morgante et al., 2006). Encouraging evidence suggests that induction of M1 neuroplasticity with such techniques improves treatment outcomes in chronic stroke patients (Uy et al., 2003; Kim et al., 2006; McDonnell et al., 2007b). Several techniques can induce neuroplastic changes in humans that persist for minutes to hours after intervention, including cortical stimulation (Pascual-Leone et al., 1994; Berardelli et al., 1998), peripheral stimulation (Ridding et al., 2001; Charlton et al., 2003), and combined cortical and peripheral nerve stimulation (Stefan et al., 2000; Ridding and Taylor, 2001; Ridding and Flavel, 2006). The latter technique, termed paired associative stimulation (PAS), comprises electrical stimulation of a peripheral nerve followed by transcranial magnetic stimulation (TMS) of contralateral M1. With a 25 ms interstimulus interval between paired stimuli, $30 \mathrm{~min}$ of PAS induces

\footnotetext{
Received Jan. 16, 2008; accepted June 20, 2008.

This work was supported by Grant 349452 from the National Health and Medical Research Council (NHMRC) of Australia. It forms a component of the PhD studies of M.V.S., who is supported by a NHMRC Dora Lush Biomedical Research scholarship. M.C.R. holds an NHMRC Senior Research Fellowship. Wethank Assoc. Prof. David Kennaway for the salivary cortisol analysis.

Correspondence should be addressed to Assoc. Prof. Michael A. Nordstrom, Discipline of Physiology and Research Centre for Human Movement Control, School of Molecular and Biomedical Science, The University of Adelaide, Adelaide SA 5005, Australia. E-mail: michael.nordstrom@adelaide.edu.au.

D0I:10.1523/JNEUROSCI.1963-08.2008

Copyright $\odot 2008$ Society for Neuroscience $\quad$ 0270-6474/08/288285-09\$15.00/0
}

an increase in corticomotor excitability in the target muscle that lasts for 30 min or more (Stefan et al., 2000). Because TMS activates pyramidal tract neurons indirectly (Day et al., 1987), and the afferent volley from the peripheral stimulus is known to alter corticospinal neuron excitability (Mariorenzi et al., 1991), it is thought that the near-synchronous arrival of the two inputs with PAS induces hebbian-like changes in synaptic efficacy, through a long-term potentiation (LTP)-like process (Stefan et al., 2000).

The variability in responses to PAS (Fratello et al., 2006; Sale et al., 2007) limits its usefulness for investigating pathophysiological changes in the brain, and as an adjunct to rehabilitative therapy. We recently found, inter alia, that the effectiveness and reproducibility of PAS-induced neuroplasticity was influenced by time of day of the PAS intervention (Sale et al., 2007), suggesting that PAS-induced neuroplasticity may be subject to circadian modulation. However, the experimental design did not allow direct testing of this hypothesis because a different group of subjects participated in the morning and afternoon experiments. The first aim of the present study was to provide a direct test of a time-of-day influence on M1 neuroplasticity by testing each subject with PAS at 8:00 A.M. and 8:00 P.M. on separate days.

Cortisol is a neuromodulator that could contribute to a timeof-day variation in PAS effectiveness. Plasma cortisol concentration is highest in the morning, immediately on awakening, and declines to reach a nadir $\sim 14 \mathrm{~h}$ after waking (Ranjit et al., 2005). Neuroplastic changes induced by PAS are the result of LTP-like mechanisms, and LTP is inhibited by a cortisol metabolite in rats (Dubrovsky et al., 1985). We measured salivary cortisol levels in 
the morning and evening to determine whether circadian changes in endogenous cortisol levels are associated with altered effectiveness of PAS. In a second experiment, subjects were tested at 8:00 P.M. (low endogenous cortisol levels), and circulating cortisol levels were manipulated by administration of a single oral dose of hydrocortisone (or placebo, on separate days). The results support the hypothesis that elevated circulating cortisol levels inhibit PAS-induced neuroplastic change in human M1.

\section{Materials and Methods}

Subjects

All subjects gave written informed consent before participation in the study, which was approved by the University of Adelaide Human Research Ethics Committee.

\section{Experimental arrangement}

Subjects were seated comfortably in an experimental chair with their left shoulder abducted at $\sim 45^{\circ}$ to allow the arm and hand to rest on a manipulandum. The hand was positioned such that the proximal phalanx of the left thumb rested in a metal ring attached to a load cell. The position of the load cell was adjusted so that it measured thumb abduction force. Thumb abduction force was displayed on an oscilloscope in front of the subject to provide visual feedback, and was filtered (low-pass at $50 \mathrm{~Hz}$ ) and digitized online $(2 \mathrm{kHz})$ via a CED 1401 interface (Cambridge Electronic Design), and stored on computer for offline analysis.

Surface EMG recordings from abductor pollicis brevis (APB) and first dorsal interosseous (FDI) muscle of the left hand were obtained using bipolar $\mathrm{Ag}-\mathrm{AgCl}$ electrodes in a belly-tendon montage. EMG signals were amplified 1000 times, filtered $(5-500 \mathrm{~Hz})$, digitized online $(2 \mathrm{kHz} /$ channel) via a CED 1401 interface, and stored on computer for offline analysis. EMG signals from both muscles were displayed for the subject on an oscilloscope to help them maintain EMG silence when required.

\section{Maximum voluntary contractions}

The abduction force exerted by the left thumb during a maximum voluntary contraction (MVC) was measured at the beginning of each session. Subjects were aided in the task by visual feedback of thumb abduction force displayed on an oscilloscope. Three trials were obtained in each session with at least $30 \mathrm{~s}$ rest given between each trial. The MVC force was defined as the largest thumb abduction force observed in the three trials.

\section{TMS and electrical stimulation of median nerve}

All subjects completed a TMS safety screen (Keel et al., 2001) and were excluded if there was a family history of epilepsy, or if they were taking any neuroactive drugs or had undergone neurosurgery. Monophasic TMS was applied through a figure-of-eight coil (outer diameter of each wing, $90 \mathrm{~mm}$ ) and a Bistim module (The Magstim Company), which connected two Magstim 200 magnetic stimulators (The Magstim Company). This allowed the output of both machines to be directed through the same coil. The coil was held tangentially to the skull with the handle pointing backwards and laterally at an angle of $45^{\circ}$ to the sagittal plane at the optimal scalp site to evoke a motor evoked potential (MEP) in the relaxed APB muscle of the left hand. With this coil placement, current flow was induced in a posterior-to-anterior direction in the brain. The optimal scalp position was marked with a pen, and the coil was held throughout the experiment by hand, with the position continually checked throughout the experiment. Electrical stimuli were applied to the median nerve at the wrist using a constant current stimulator (DS7 stimulator; Digitimer) with bipolar surface electrodes separated by $30 \mathrm{~mm}$, and with the cathode proximal. Stimuli were square waves with a pulse width of $200 \mu$ s.

\section{PAS}

The PAS protocol used in the present study has been described previously (Sale et al., 2007). PAS involves a series of paired peripheral and cortical stimuli. An electrical stimulus was delivered to the median nerve of the left wrist at an intensity sufficient to produce a small motor response in APB ( $\sim 300 \%$ of perceptual threshold). The electrical stimulus was followed $25 \mathrm{~ms}$ later by suprathreshold TMS over the hand area of the right motor cortex. TMS intensity was established before PAS and produced a
MEP in resting APB of $0.5-1.0 \mathrm{mV}$ (this intensity is termed $\mathrm{SI}_{\text {pre }}$ ). For PAS, 90 paired peripheral and cortical stimuli were delivered at a frequency of $0.05 \mathrm{~Hz}$ (duration $30 \mathrm{~min}$ ).

The subject's attentional focus is an important factor influencing PAS effectiveness (Stefan et al., 2004). Therefore, subjects were instructed to direct their attention onto the stimulated (left) hand during the PAS intervention. To quantify this, subjects received intermittent weak (200\% perceptual threshold) electrical stimuli to their left thumb via ring electrodes (Stefan et al., 2004). Between 7 and 10 stimuli were applied at random intervals throughout the $30 \mathrm{~min}$ PAS session, and subjects were instructed to count and remember the number of thumb stimuli they received. When a thumb stimulus was delivered, it occurred at the midpoint of the interval between successive paired stimuli in the PAS protocol. After the PAS protocol had concluded, subjects were asked to report the number of stimuli delivered to the thumb. An error score was calculated, which was the difference between the number of stimuli delivered to the thumb and the number reported by the subject.

\section{Experiment 1: effect of time of day and endogenous cortisol levels} on PAS effectiveness

Twenty-five subjects ( 11 male, 14 female; aged $20-48$ years; mean $27 \pm$ 2 years) participated in this study. Each subject was tested on two separate occasions, once in the morning (8:00 A.M.) and once in the evening (8 P.M.). The order of the two sessions was randomized and the sessions were separated by at least 1 week. During the morning (A.M.) session, PAS commenced at 8:33 A.M. \pm 2 min, and in the evening (P.M.) session, PAS commenced at 8:15 P.M. \pm 2 min. Subjects had been awake $126 \pm 6$ min before the commencement of PAS in the morning experiments, and $766 \pm 13 \mathrm{~min}$ in the evening experiments. Subjects were instructed to have a light meal before both experiments. The last meal was consumed $98 \pm 6$ min before the commencement of PAS in the morning experiments, and $107 \pm 5 \mathrm{~min}$ in the evening experiments, an insignificant difference (unpaired $t$ test).

\section{Single-pulse and paired-pulse TMS measures of motor} cortex excitability

Several measures of motor cortex excitability were made before and after the PAS intervention during each experimental session (see Fig. $1 A$ for time line of experimental assessments).

Resting motor threshold (RMT) was defined as the minimum stimulus intensity required to evoke a MEP in relaxed $\mathrm{APB}$ of $>50 \mu \mathrm{V}$ in three of five consecutive trials (Carroll et al., 2001). Active motor threshold (AMT) was defined as the minimum stimulus intensity required to evoke a MEP of $>200 \mu \mathrm{V}$ in the active (5\% MVC) APB in three of five consecutive trials. The stimulus intensity was expressed as a percentage of maximum stimulator output (\% MSO). RMT was assessed before and after PAS in all experiments. AMT was assessed before PAS only in experiment 1 (see below). All TMS pulses were delivered using the output of the Bistim module, which reduces the output by $\sim 10 \%$ compared with a single Magstim and leads to higher threshold values as \% MSO.

Mean peak-to-peak amplitude of the APB and FDI MEP at rest was calculated by averaging the individual peak-to-peak amplitudes of MEPs elicited by 20 TMS pulses $\left(0.2 \mathrm{~s}^{-1}, \mathrm{SI}_{\text {pre }}\right)$ delivered immediately before (pre-PAS), and 5 min after PAS (post-PAS).

We assessed cortical inhibition because GABAergic systems influence LTP in rat motor cortex (Hess and Donoghue, 1994) and plasticity in human M1 (Butefisch et al., 2000; McDonnell et al., 2007a). The cortical silent period (CSP) duration for APB was assessed with a TMS intensity of $130 \%$ RMT and a contraction strength of $15 \%$ MVC. Subjects were given visual feedback of their force level via an oscilloscope. Ten TMS pulses were delivered at a frequency of $0.2 \mathrm{~Hz}$. The duration of the APB CSP was measured off-line from the rectified EMG traces for each trial and averaged for the 10 trials. CSP duration was defined as the time from TMS onset until the resumption of continuous EMG traces at prestimulus levels.

Short-interval intracortical inhibition (SICI) was assessed using a protocol similar to that described previously (Kujirai et al., 1993). Pairedpulse TMS was delivered at an interstimulus interval of $3 \mathrm{~ms}$. The first (conditioning) stimulus was subthreshold for producing a MEP in active 


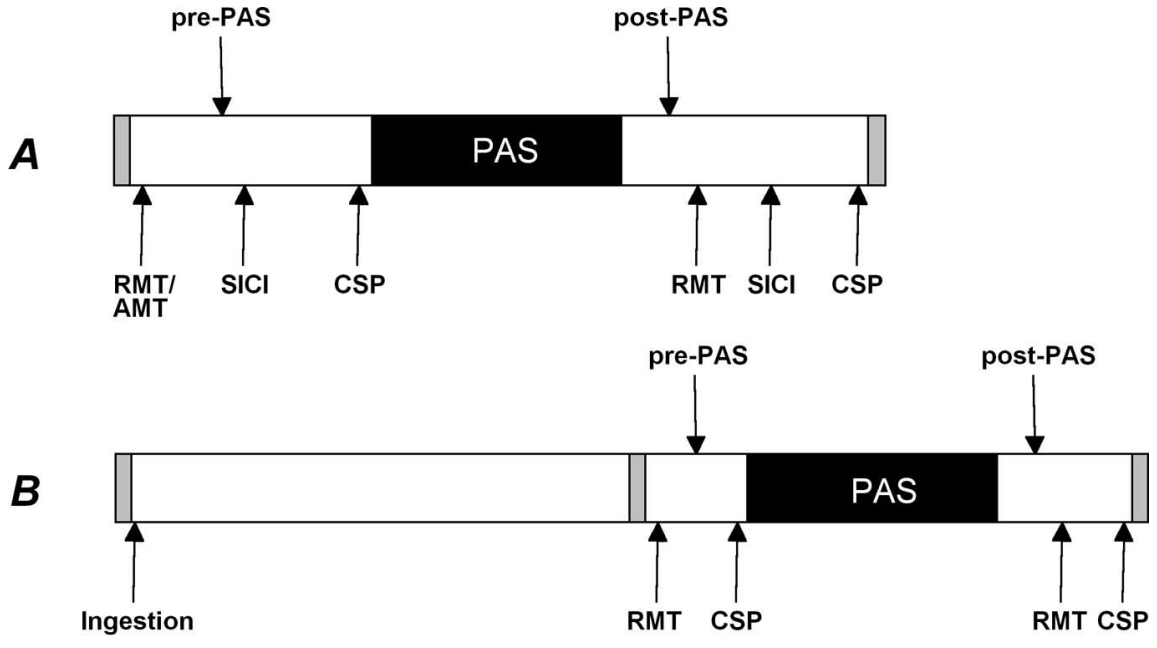

Figure 1. $\boldsymbol{A}, \boldsymbol{B}$, Schematic diagram of the testing protocol indicating the approximate relative timings for assessment of neurophysiological parameters before and after PAS in experiment $1(\boldsymbol{A})$ and experiment $2(\boldsymbol{B})$. The gray bars indicate when saliva samples were collected. Measurements of APB MEP amplitude were made at two time points: pre-PAS and post-PAS. Ingestion, Oral dose of hydrocortisone or placebo.

$\mathrm{APB}$, and activated intracortical inhibitory circuits that reduced the size of the MEP elicited by the suprathreshold test stimulus delivered $3 \mathrm{~ms}$ later. Four conditioning intensities were used: $60 \%, 70 \%, 80 \%$, and $90 \%$ $\mathrm{AMT}$. The test stimulus intensity was set at $\mathrm{SI}_{\text {pre }}$ before PAS. Because the extent of SICI may be influenced by the size of the test MEP, the test TMS intensity was adjusted post-PAS to match the amplitude of the test MEP with that seen pre-PAS. This TMS intensity was termed SI $_{\text {post }}$. The effect of conditioning TMS intensity on MEP amplitude was assessed in separate blocks. Each block consisted of 20 trials $\left(0.2 \mathrm{~s}^{-1}\right)$, which included 10 paired stimuli and 10 test-alone stimuli in pseudorandom order. The order of testing blocks containing each conditioning intensity was randomized. The size of the conditioned MEP was expressed as a percentage of the unconditioned MEP in each block to quantify the effectiveness of SICI.

\section{Salivary cortisol assay}

Saliva samples were collected from each subject before commencement of PAS (pre-PAS) and at the end of each experiment (post-PAS). Saliva was frozen at $-20^{\circ} \mathrm{C}$ until assayed. On the day of assay, the saliva samples were thawed and centrifuged. Twenty-five microliters of saliva were assayed in duplicate for cortisol by ELISA (HS-Cortisol; Salimetrics).

\section{Experiment 2: exogenous cortisol and PAS effectiveness}

Seventeen subjects ( 8 male, 9 female, aged 19-35 years; mean $24 \pm 1$ years) participated in a randomized, double-blind, placebo-controlled study to determine whether a single oral dose of hydrocortisone modulates PAS effectiveness (referred to hereinafter as the exogenous cortisol study). One subject complained of a mild headache (hydrocortisone session), but completed the experiment. One additional subject was tested but withdrew because of feeling unwell (in placebo session).

Subjects were tested at 8:00 P.M. (when endogenous cortisol is low) on two occasions separated by at least 1 week. Subjects received a gelatincased capsule containing either hydrocortisone (Hysone 24 mg; Alphapharm) or starch (order randomized for sessions 1 and 2), which was consumed with a glass of low-fat milk 60 min before the commencement of the experiment. Subjects were instructed to fast for $3 \mathrm{~h}$ before the commencement of the experiment. The last meal was consumed $195 \pm$ $18 \mathrm{~min}$ before the commencement of PAS in the placebo experiments, and $199 \pm 41 \mathrm{~min}$ in the cortisol experiments, an insignificant difference (unpaired $t$ test). PAS commenced at 8:09 P.M. $\pm 2 \mathrm{~min}$ in the placebo session and 8:08 P.M. $\pm 1 \mathrm{~min}$ in the cortisol session. Subjects had been
10 mins

awake for $738 \pm 27 \mathrm{~min}$ in the placebo session, and $737 \pm 32 \mathrm{~min}$ in the cortisol session, an insignificant difference (unpaired $t$ test). Saliva samples were taken before ingestion of medication (pre-ingestion), before PAS (pre-PAS), and after PAS (post-PAS), and later assayed for salivary cortisol concentration.

The experimental procedures and protocol for the exogenous cortisol study were as for experiment 1, except that AMT and SICI were not assessed (see Fig. $1 B$ for time line of experimental procedures).

\section{Statistical analysis}

Experiment 1. Three-way repeated-measures ANOVA was performed on MEP amplitude data from APB and FDI with within-subject factors intervention (two levels: pre-PAS and postPAS), time of day (two levels: A.M. and P.M.), and between-subject factor gender (two levels: male and female) to determine the effect of PAS and time of day on the extent of MEP facilitation. Two-way repeated-measures ANOVA assessed the effect of intervention and time of day on APB resting motor threshold and cortical silent period. One-way repeated-measures ANOVA assessed the effect of time of day on APB active motor threshold. Three-way repeated-measures ANOVA with withinsubject factors of intervention, time of day, and conditioning intensity (four levels: 60\% AMT, 70\% AMT, 80\% AMT, and $90 \%$ AMT) assessed the effect of these factors on SICI.

Experiment 2. Three-way repeated-measures ANOVA was performed on MEP amplitude data from APB and FDI with within-subject factors intervention (two levels: pre-PAS and post-PAS) and medication (two levels: hydrocortisone and placebo), and gender (two levels: male and female) to determine the effect of PAS and medication on the extent of MEP facilitation. Two-way repeated-measures ANOVA assessed the effect of intervention and medication on APB resting motor threshold and cortical silent period.

Linear regression analysis was used on data from experiments 1 and 2 to examine the relationship between salivary cortisol concentration and both APB MEP facilitation (post-PAS MEP amplitude/pre-PAS MEP amplitude) and the pre-PAS cortical silent period duration. The salivary cortisol concentration data were log-transformed to improve homoschedasticity. The strength of the relationship was quantified by the coefficient of determination $\left(r^{2}\right)$.

For all analyses, $p<0.05$ was chosen as the significance level, and unless stated otherwise, all group data are reported as mean \pm SEM. Fisher's PLSD post hoc tests were performed as appropriate.

\section{Results}

Experiment 1: effect of time of day and endogenous cortisol levels on PAS effectiveness

TMS intensity used during PAS ( $\mathrm{SI}_{\text {pre }}$ ) was not significantly different in the endogenous cortisol experiments between the morning and evening sessions ( $76 \pm 3 \% \mathrm{MSO}$ vs $74 \pm 3 \% \mathrm{MSO}$, respectively), corresponding to $127 \pm 3 \% \mathrm{RMT}$ versus $127 \pm 3 \%$ RMT. Intensity of peripheral nerve stimulation during PAS was $6.5 \pm 0.5 \mathrm{~mA}$ for the morning session and $5.8 \pm 0.5 \mathrm{~mA}$ for the evening session, an insignificant difference (unpaired $t$ test).

ANOVA revealed no effect of time of day $\left(F_{(1,24)}=2.14\right)$ or intervention $\left(F_{(1,24)}=1.65\right)$ on resting motor threshold in the endogenous cortisol experiments. In the morning, RMT was $60 \pm 2 \%$ MSO pre-PAS and $59 \pm 2 \%$ MSO post-PAS. In the evening, RMT was $59 \pm 2 \%$ MSO pre-PAS and $57 \pm 3 \%$ MSO post-PAS. There was also no significant effect of time of day on active motor threshold $\left(F_{(1,24)}=3.14\right)$. AMT was only assessed 


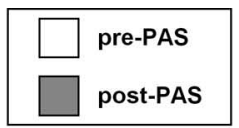

A

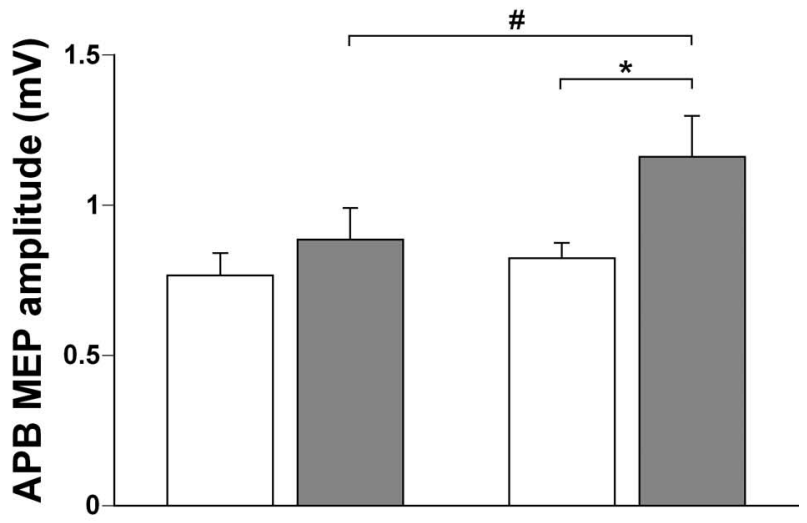

$B$

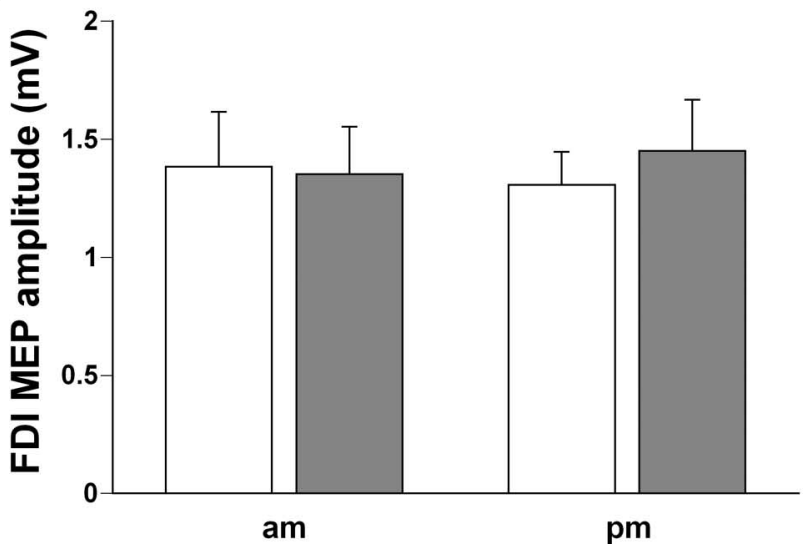

Figure 2. $\boldsymbol{A}, \boldsymbol{B}$, Group (mean \pm SEM) MEP amplitude for APB $(\boldsymbol{A})$ and FDI $(\boldsymbol{B})$ before (pre$P A S$ ) and after (post-PAS) paired associative stimulation. Data are from morning experiments (am) on the left, and evening (pm) experiments on the right. APB MEP amplitude was significantly larger post-PAS than pre-PAS in the evening session ( ${ }^{*} p<0.001$ ), but not in the morning. APB MEP amplitude post-PAS was significantly greater in the evening than in the morning $\left({ }^{\sharp} p<0.001\right)$. FDI MEP amplitude $(\boldsymbol{B})$ was not affected by PAS or time of day.

before PAS. In the morning AMT was $48 \pm 2 \% \mathrm{MSO}$ and in the evening, $46 \pm 2 \%$ MSO.

The extent of MEP facilitation induced in APB by PAS was larger in the evening sessions than in the morning, and this effect was consistent across gender $\left(F_{(1,23)}=0.40\right)$; thus, the data were pooled for males and females. The group data on PAS effectiveness for the 25 subjects are summarized in Figure 2. On average, APB MEP amplitude increased by $15 \%$ in morning experiments and by $41 \%$ in evening experiments (Fig. 2A). There was a significant effect of time of day $\left(F_{(1,24)}=7.67, p=0.011\right)$ and intervention $\left(F_{(1,24)}=9.84, p=0.005\right)$ on APB MEP amplitude. There was also a time of day $\times$ intervention interaction $\left(F_{(1,24)}=4.45\right.$, $p=0.045)$, indicating that the effectiveness of PAS was influenced by the time of day. Post hoc analysis revealed that there was significant APB MEP facilitation in the evening (Fisher, $p<$ 0.001), whereas there was no significant change in APB MEP amplitude after PAS in the morning (Fisher). APB MEP amplitude before PAS was not significantly different between sessions (A.M., $0.76 \pm 0.07 \mathrm{mV}$; P.M., $0.78 \pm 0.06 \mathrm{mV}$ ). APB MEP amplitude after PAS (post-PAS) was significantly greater in the

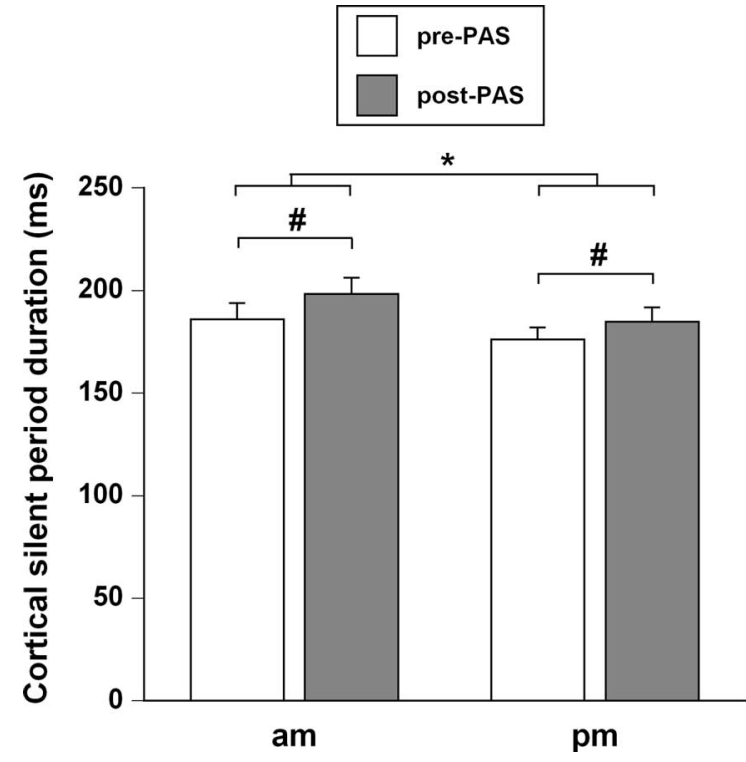

Figure 3. Group (mean \pm SEM) data of cortical silent period duration before (pre-PAS) and after (post-PAS) paired associative stimulation. Data from morning experiments (am) are on the left, and evening (pm) experiments are on the right. Cortical silent period duration was significantly longer in the morning ( ${ }^{*} p=0.006$ ) than in the evening. There was a significant increase in cortical silent period duration after PAS in both the morning and evening sessions $\left({ }^{\#} p=\right.$ $0.002)$

evening compared with the morning ( $p<0.001$ ) (Fig. 2A). As expected, PAS did not result in significant facilitation of MEPs evoked in the FDI muscle (intervention, $F_{(1,24)}=0.95$ ). The FDI MEP was 3\% smaller after PAS in the morning, and 16\% larger after PAS in the evening (Fig. 2B). There was no significant effect of time of day on FDI MEP amplitude $\left(F_{(1,24)}=0.76\right)$.

Time of day did not influence attention-related error scores, indicating that the subjects were attending to the task equally well in the morning and evening experiments. The mean error score in the morning session was $0.24 \pm 0.10$ and in the evening session, $0.28 \pm 0.09\left(F_{(1,24)}=0.07\right)$.

Cortical silent period data from experiment 1 are summarized in Figure 3. Both time of day $\left(F_{(1,24)}=9.04, p=0.006\right)$ and intervention $\left(F_{(1,24)}=27.47, p<0.001\right)$ influenced cortical silent period duration. The interaction term was not significant $\left(F_{(1,24)}\right.$ $=0.28)$, indicating that PAS had similar effects on the cortical silent period in morning and evening experiments. Cortical silent period duration was significantly shorter in the evening (prePAS, $183 \pm 5 \mathrm{~ms}$ ) compared with the morning (pre-PAS, $193 \pm$ $7 \mathrm{~ms})(p=0.016)$, and the duration of the silent period increased by $\sim 6 \%$ after PAS (pre-PAS, $188 \pm 4 \mathrm{~ms}$ vs post-PAS $199 \pm 4 \mathrm{~ms}$; $p=0.002$ ).

PAS did not significantly alter SICI (intervention, $F_{(1,72)}=$ 0.30; see Fig. 4), and the overall effectiveness of SICI was similar in the morning and evening (time of day, $F_{(1,72)}=1.29$ ). As expected, SICI was a function of conditioning TMS intensity $\left(F_{(3,72)}=25.76, p<0.001\right)$. Post hoc analysis revealed that MEP suppression with a conditioning intensity of $60 \%$ AMT was significantly less than MEP suppression using all other conditioning intensities (Fisher, $p<0.001$ ), and that MEP suppression with a conditioning intensity of $70 \%$ AMT was significantly less than MEP suppression using conditioning intensities of $80 \%$ AMT and $90 \%$ AMT (Fisher, $p=0.001$ ). Conditioning at 60\% AMT did not suppress the MEP (paired $t$ test), whereas higher conditioning intensities produced significant inhibition of the MEP 
A

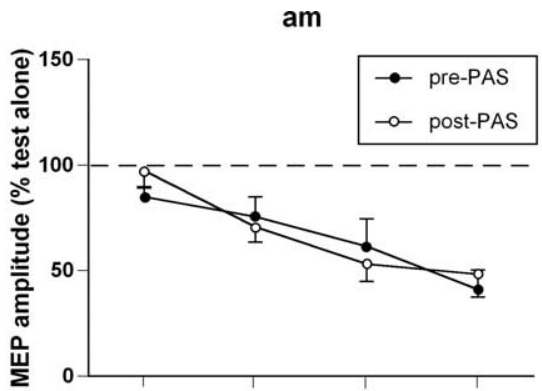

pre-PAS

B

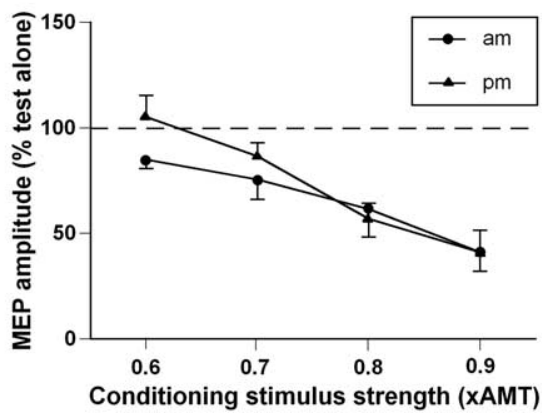

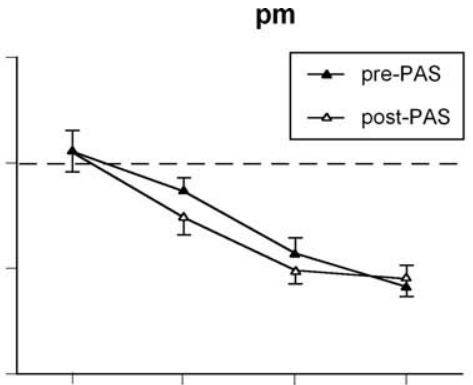

post-PAS

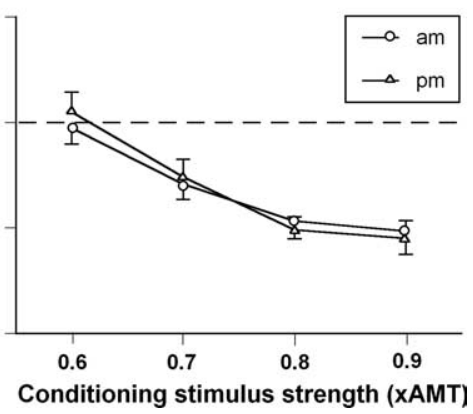

hydrocortisone, APB MEP amplitude decreased (non-significantly) by 6\% (Fisher). FDI MEP amplitude (Fig. 5B) was not influenced by medication $\left(F_{(1,16)}=3.77\right)$ or intervention $\left(F_{(1,16)}=1.98\right)$, and the interaction term was not significant $\left(F_{(1,16)}=\right.$ 0.35).

Cortical silent period data from experiment 2 are summarized in Figure 6. Both medication $\left(F_{(1,16)}=5.16, p=0.037\right)$ and intervention $\left(F_{(1,16)}=216.37, p<0.001\right)$ influenced cortical silent period duration. The interaction term was not significant $\left(F_{(1,16)}=2.28\right)$, indicating that PAS had similar effects on the cortical silent period in hydrocortisone and placebo sessions. Cortical silent period duration was significantly shorter in the placebo (pre-PAS, $193 \pm 5 \mathrm{~ms}$ ) compared with the hydrocortisone session (pre-PAS, $206 \pm 3 \mathrm{~ms})(p<$ 0.001 ), and the duration of the silent period increased by $\sim 10 \%$ after PAS (prePAS, $199 \pm 3 \mathrm{~ms}$ vs post-PAS $218 \pm 3 \mathrm{~ms}$ $(p<0.001)$.

Figure 4. Influence of PAS and time of day on SICl. Group (mean \pm SEM) SICI data from 20 subjects assessed in the morning (am; circles) and evening (pm; triangles), before (pre-PAS; filled symbols) and after (post-PAS; open symbols) paired associative stimulation. SICl was assessed with paired-pulse TMS using four different conditioning TMS intensities (60-90\% AMT), with an interstimulus interval of $3 \mathrm{~ms}$. SICI was quantified as percentage of MEP amplitude obtained in conditioned trials compared with test-alone trials. SICI was unchanged after PAS both in the A.M. and P.M.. The overall level of SICI did not differ between A.M. and P.M. sessions.

(paired $t$ tests, $p<0.001$ ). The APB MEP amplitude in singlepulse trials did not differ pre- and post-PAS (intervention, $F_{(1,72)}$ $=4.17)$, because of a small reduction of test TMS intensity postPAS (76\% MSO pre-PAS vs $74 \%$ MSO post-PAS). There was also no significant effect of time of day $\left(F_{(1,72)}=0.01\right)$ on MEP amplitude in single-pulse trials. This indicates that test MEP amplitudes were well matched throughout the assessment of SICI.

\section{Experiment 2: Exogenous cortisol and PAS effectiveness}

TMS intensity used during PAS ( $\mathrm{SI}_{\text {pre }}$ ) was not significantly different between the placebo and cortisol sessions (75 $\pm 3 \% \mathrm{MSO}$ vs $75 \pm 3 \%$ MSO, respectively), corresponding to $123 \pm 2 \%$ RMT versus $123 \pm 3 \%$ RMT. The intensity of peripheral nerve stimulation during PAS was $6.7 \pm 0.6 \mathrm{~mA}$ for the placebo session and $6.5 \pm 0.5 \mathrm{~mA}$ for the cortisol session, an insignificant difference (unpaired $t$ test).

ANOVA revealed no significant effect of medication $\left(F_{(1,16)}=\right.$ $0.48)$ or intervention $\left(F_{(1,16)}=0.01\right)$ on resting motor threshold. In the placebo session, RMT was $61 \pm 2 \%$ MSO pre-PAS and $61 \pm 2 \%$ MSO post-PAS. In the cortisol session, RMT was $61 \pm$ $2 \%$ MSO pre-PAS and $60 \pm 2 \%$ MSO post-PAS.

There was significant facilitation of APB MEP amplitude after PAS in the placebo session, but not in the session when subjects received oral hydrocortisone (Fig. $5 A$ ). This effect was consistent across gender $\left(F_{(1,15)}=0.21\right)$, and thus data were pooled for males and females. Two-way repeated-measures ANOVA indicated no significant effect of medication $\left(F_{(1,16)}=0.64\right)$ or intervention $\left(F_{(1,16)}=3.28\right)$, but there was a significant medication $\times$ intervention interaction $\left(F_{(1,16)}=9.78, p=0.007\right)$, indicating that the effect of PAS on MEP amplitude was influenced by medication. APB MEP amplitude increased significantly by $28 \%$ in the placebo session after PAS (Fisher, $p=0.039$ ), yet with oral

\section{Salivary cortisol levels and relationships with PAS effectiveness and cortical silent period}

Saliva samples obtained during the experiments were assayed for salivary cortisol concentration. For experiment 1, saliva samples were taken before (pre-PAS) and after PAS (post-PAS). There was a significant effect of time of day $\left(F_{(1,24)}=86.90, p<\right.$ $0.001)$ and intervention $\left(F_{(1,24)}=10.72, p=0.003\right)$ on salivary cortisol concentration (Fig. $7 A$ ). There was also a significant interaction of time of day $\times$ intervention $\left(F_{(1,24)}=5.57, p=0.027\right)$. Post hoc analysis revealed that salivary cortisol concentration before PAS in the morning session was higher than at all other times of saliva sampling $(p<0.001)$. Salivary cortisol concentration after PAS (post-PAS) in the morning session was greater than salivary cortisol concentration in the evening sessions $(p<$ 0.001) (Fig. 7A).

For experiment 2, saliva samples were taken before ingestion of the medication (pre-ingestion) as well as before (pre-PAS) and after PAS (post-PAS). There was a significant effect of time (three levels: pre-ingestion, pre-PAS, post-PAS) $\left(F_{(2,32)}=5.07, p=\right.$ $0.012)$ and medication $\left(F_{(1,32)}=11.46, p=0.004\right)$ on salivary cortisol levels (Fig. $7 B$ ). There was also a significant time $\times$ medication $\left(F_{(1,32)}=4.78, p=0.015\right)$ interaction. Post hoc analysis revealed that oral hydrocortisone administration elevated salivary cortisol levels, with both pre-PAS and post-PAS cortisol concentrations significantly higher than pre-ingestion levels $(p<0.001)$. There was no significant difference in salivary cortisol concentration during the placebo experiments.

Because salivary cortisol concentration changed over the time it took to deliver PAS (Fig. 7A), the pre-PAS and post-PAS salivary cortisol concentrations were averaged to provide a value that reflects the mean circulating cortisol level during PAS. This was used in the linear regression analysis of the association between salivary cortisol level and MEP facilitation induced by PAS (Fig. $7 C, D$ ), and cortical silent period duration (Fig. $7 E, F)$. For experiment 1 , there was no significant relationship between the $(\log )$ average salivary cortisol concentration and the extent of APB 


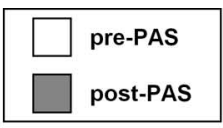

A \#

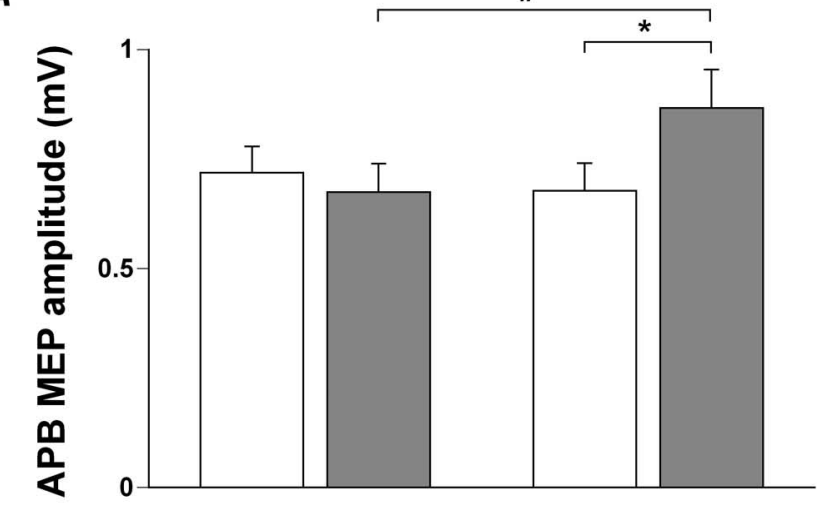

B

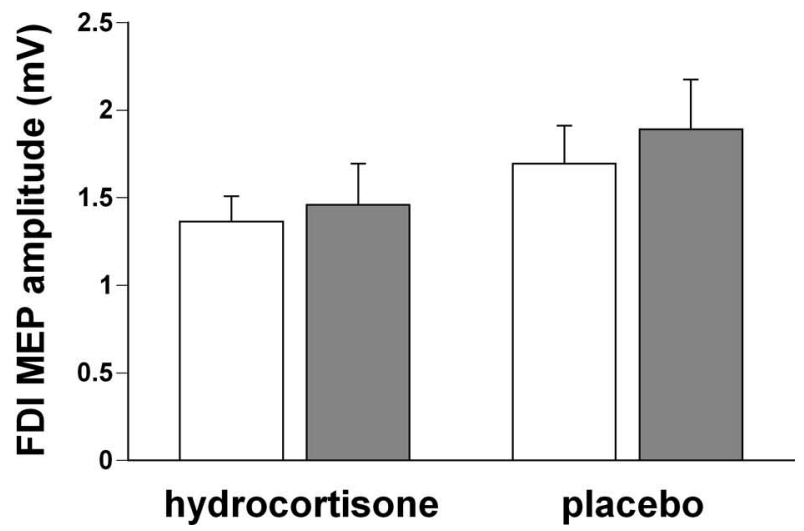

Figure 5. $\boldsymbol{A}, \boldsymbol{B}$, Group (mean \pm SEM) MEP amplitude for APB $(\boldsymbol{A})$ and FDI $(\boldsymbol{B})$ before (pre$\mathrm{PAS}$ ) and after (post-PAS) paired associative stimulation. Data from the hydrocortisone (cortisol) administration session are on the left, and data from the placebo session are on the right. APB MEP amplitude was significantly larger post-PAS than pre-PAS in the placebo session $\left({ }^{*} p=0.007\right)$, but not in the cortisol session. APB MEP amplitude post-PAS was significantly greater in the placebo than in the cortisol session $\left({ }^{\#} p=0.039\right)$. FDI MEP amplitude $(\boldsymbol{B})$ was not affected by PAS or medication.

MEP facilitation $\left(r^{2}=0.04\right)$ (Fig. 7C). In contrast, with exogenous cortisol administration in experiment 2 , there was a weak but significant negative relationship between the average salivary cortisol concentration and the extent of APB MEP facilitation after PAS $\left(r^{2}=0.13, p=0.039\right)$ (Fig. $\left.7 D\right)$. There was greater facilitation of the APB MEP after PAS when salivary cortisol concentration was low. There was no significant relationship between average salivary cortisol concentration and pre-PAS cortical silent period duration in experiment $1\left(r^{2}=0.002\right)$ (Fig. $7 E$ ). However, in experiment 2 , there was a significant positive relationship between salivary cortisol concentration and pre-PAS cortical silent period duration $\left(r^{2}=0.14, p=0.028\right)$ (Fig. 7F). There was no significant relationship between the pre-PAS CSP values and MEP facilitation ratio for either experiment $1\left(r^{2}=\right.$ $0.02, n=50)$ or experiment $2\left(r^{2}=0.08, n=34\right)$ (data not shown).

\section{Discussion}

The principal novel findings from this study are that MEP facilitation after PAS was influenced by time of day and oral hydrocortisone administration. There was significant MEP facilitation in the target muscle after PAS in the evening. However, during

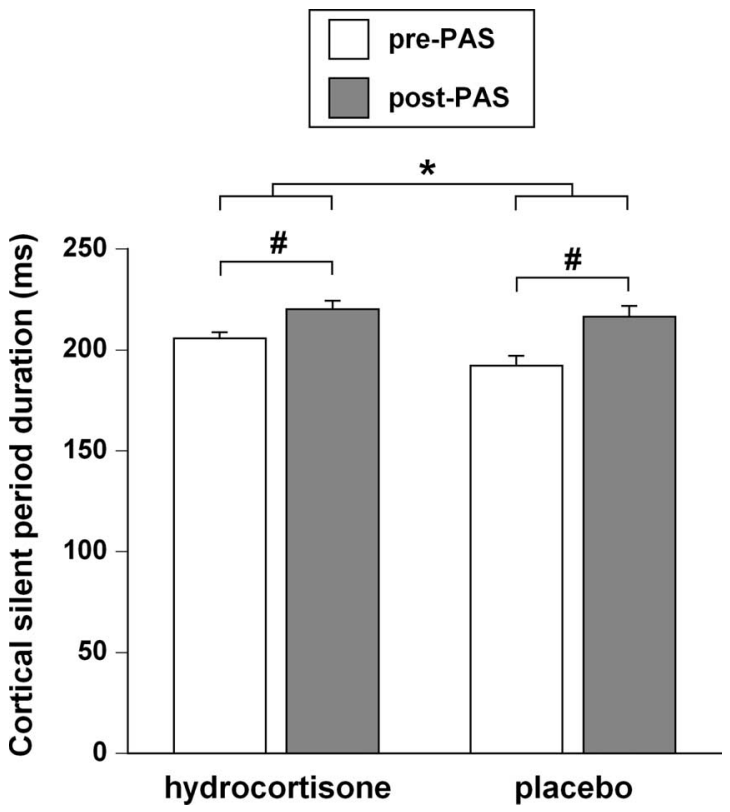

Figure 6. Group (mean \pm SEM) data of cortical silent period duration before (pre-PAS) and after (post-PAS) paired associative stimulation. Data from the hydrocortisone (cortisol) administration group are on the left, and data from the placebo group are on the right. Cortical silent period duration was significantly longer in the cortisol ( $\left.{ }^{*} p<0.001\right)$ than in the placebo group. There was a significant increase in cortical silent period duration after PAS in both sessions $\left({ }^{\#} p<0.001\right)$.

periods of high circulating cortisol levels (in the morning or with oral hydrocortisone in the evening), there was no significant MEP facilitation. Additionally, the time of day, PAS, and circulating cortisol levels influenced CSP duration.

\section{M1 neuroplasticity is influenced by time of day}

We recently reported that PAS was more effective in experiments conducted in the afternoon than in the morning (Sale et al., 2007); however, different subjects were tested at those times. The present study extends that result by showing, in the same group of subjects, that PAS effectiveness is greater in the evening than in the morning.

PAS induces M1 plasticity by LTP-like mechanisms (Stefan et al., 2000, 2002; Wolters et al., 2003, Ziemann et al., 2004). M1 is considered the primary site of corticomotor excitability change induced by PAS (Stefan et al., 2000; Wolters et al., 2003; Müller et al., 2007), but we have not excluded effects on remote sites such as spinal cord or ipsilateral primary sensory cortex (Murakami et al., 2008). PAS-induced changes in spinal excitability have been reported (Meunier et al., 2007), although others find no change (Stefan et al., 2000; Wolters et al., 2003).

It has been proposed that wakefulness favors LTP-like processes and leads to net synaptic potentiation, whereas sleep favors LTD-like processes and leads to homeostatic synaptic depression (Tononi and Cirelli, 2006). According to this theory, prolonged wakefulness should make induction of LTP-like plasticity more difficult, because LTP can be saturated by previous LTP-like learning (Rioult-Pedotti et al., 2000; Whitlock et al., 2006). LTP in rat cerebral cortex is induced more readily 1-60 min after sleep than after several hours of wakefulness (Vyazovskiy et al., 2008). In contrast, we found PAS more effective in the evening, when subjects had been awake $766 \pm 13 \mathrm{~min}$, compared with the morning when they had been awake $126 \pm 6 \mathrm{~min}$. The reasons for this 

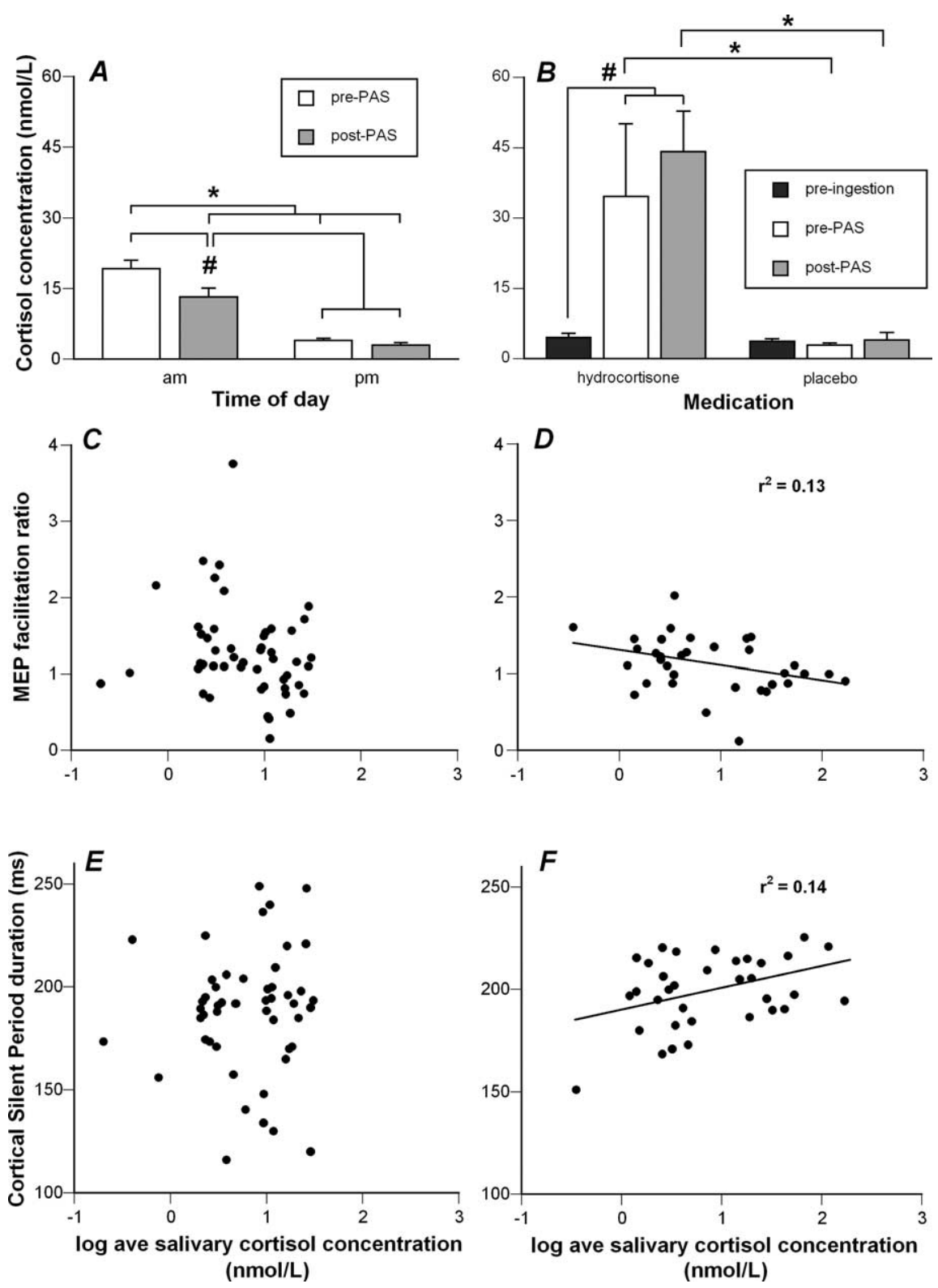

Figure 7. $A-F$, Salivary cortisol concentration $(\boldsymbol{A}, \boldsymbol{B})$ and the relationship between salivary cortisol concentration and APB MEP facilitation after $\operatorname{PAS}(\boldsymbol{C}, \boldsymbol{D})$ and cortical silent period duration $(\boldsymbol{E}, \boldsymbol{F})$. Data from study 1 (endogenous cortisol) are on the left, and data from study 2 (hydrocortisone administration) are on the right. Pre-PAS salivary cortisol concentration in the morning was significantly greater than all other samples $(\boldsymbol{A})\left({ }^{*} p<0.001\right)$. Post-PAS salivary cortisol concentration in the morning was significantly greater than both P.M. samples, but less than the pre-PAS A.M. sample ( $\left.{ }^{\#} p<0.001\right)$. $\boldsymbol{B}$, Exogenous cortisol administration significantly elevated salivary cortisol concentration in the evening compared with placebo $\left.{ }^{*} p<0.001\right)$. $C$ Linear regression analysis revealed a nonsignificant relationship $\left(r^{2}=0.04\right)$ between the $\log$ of average salivary cortisol concentration and APB MEP facilitation ratio (post-PAS MEP amplitude/pre-PAS MEP amplitude) in study 1 (endogenous cortisol). $\boldsymbol{D}$, Linear regression analysis revealed a significant negative relationship $\left(r^{2}=0.13, p=0.039\right)$ between the log of average salivary cortisol concentration and APB MEP facilitation ratio in study 2 (hydrocortisone administration). E, Nonsignificant relationship $\left(r^{2}\right.$ $=0.002$ ) between the $\log$ of average salivary cortisol concentration and pre-PAS cortical silent period duration in study $1 . \boldsymbol{F}$, Significant relationship $\left(r^{2}=0.14, p=0.028\right)$ between the $\log$ of average salivary cortisol concentration and pre-PAS cortical silent period duration in study 2.

discrepancy are not clear, but our results suggest that other factors, such as cortisol levels, influence LTP-like neuroplasticity.

\section{Cortisol and M1 neuroplasticity}

Time-of-day modulation of PAS effectiveness may be due to circadian changes in neuromodulators. LTP in hippocampus exhibits a circadian rhythm (Raghavan et al., 1999; Chaudhury et al., 2005). Cortisol is a candidate neuromodulator because it has a circadian rhythm, and a corticosterone metabolite impairs LTP in rat hippocampus (Dubrovsky et al., 1985). Acute (Newcomer et al., 1999; de Quervain et al., 2000) and chronic (Starkman et al., 1992) elevation of plasma cortisol have been associated with memory impairment in humans.

Plasma cortisol concentration rises quickly after awakening in the morning, and is low during the afternoon and evening (Ranjit et al., 2005). PAS effectiveness is enhanced in the evening (Fig. 2) when endogenous cortisol is low. Effects of PAS in the evening are blocked by a single oral dose of hydrocortisone (Fig. 5), which is metabolized to cortisol in the body.

Cortisol has wide-ranging effects on physiological processes, and we are unable to determine whether impairment of neuroplasticity by hydrocortisone is mediated directly by cortisol or indirectly by effects on other neuromodulator(s). Cortisol may also delay the time course of PAS effects, rather than reducing them. Future studies could address this by assessing MEP facilitation at multiple time points after PAS.

Cortisol is rapidly metabolized on release (Edwards et al., 2001), and fluctuations in cortisol levels during experiment 1 (Fig. 7A) may contribute to the lack of association between salivary cortisol levels and PAS effectiveness. Higher levels of cortisol in experiment 2 (Fig. 7B) may be more effective in suppressing neuroplasticity and contribute to the significant negative association between salivary cortisol and neuroplasticity in experiment 2. A single oral dose of hydrocortisone impairs learning and memory in humans (de Quervain et al., 2000; Hsu et al., 2003), and salivary cortisol levels in experiment 2 are in the range reported by de Quervain et al. (2000).

Although circulating cortisol levels appear to influence PAS-induced neuroplasticity, the weak correlation between these variables indicates a contribution from other factors. Because experiment 1 was performed at two different times of day, circadian modulation of other (nonmeasured) neuromodulators may have influenced results, including dopamine (Castañeda et al., 2004) and melatonin (Collins and Davies, 1997; El-Sherif et al., 2003), both of which influence LTP (Collins and Davies, 1997; Kusuki et al., 1997; El-Sherif et al., 2003). Because both sessions of experiment 2 were in the evening, between-session circadian variation in other neuromodulators would have less influence on PAS effectiveness in experiment 2 than in experiment 1.

Subjects participated in two experimental sessions separated by at least 1 week. Between-session changes in factors known to modulate cortical excitability, including physical activity (Cot- 
man and Engesser-Cesar, 2002), may have influenced the results. Changes in menstrual cycle hormones also influence cortical excitability (Smith et al., 1999); however, we found no gender differences in PAS-induced neuroplasticity. Menopausal women aged $>50$ years are less responsive to PAS according to Tecchio et al. (2008); however, that study reported no differences between young men and women, consistent with the present study.

\section{Modulation of intracortical inhibition}

GABAergic inhibitory tone modulates LTP-like plasticity, and we considered whether changes in M1 inhibitory tone influenced PAS effectiveness. Downregulation of $\mathrm{GABA}_{\mathrm{A}}$-mediated inhibition promotes LTP induction in rat motor cortex slices (Hess and Donoghue, 1994) and PAS-induced neuroplasticity in human M1 (Butefisch et al., 2000). Additionally, the $\mathrm{GABA}_{\mathrm{B}}$ receptor agonist baclofen suppresses LTP-like plasticity in human M1 (McDonnell et al., 2007a). GABA ${ }_{\mathrm{B}}$ inhibitory tone in M1, as assessed by CSP, was higher in morning sessions and with evening hydrocortisone administration. This is when PAS was less effective, suggesting that $\mathrm{GABA}_{\mathrm{B}}$ inhibitory tone may modulate PAS effectiveness, consistent with findings obtained with baclofen (McDonnell et al., 2007a). However, the absence of a significant relationship between pre-PAS CSP duration and MEP facilitation ratio in experiment 1 and experiment 2 argues against a direct effect of physiological variation in $\mathrm{GABA}_{\mathrm{B}}$ inhibitory tone on PAS effectiveness.

A prolonged CSP after PAS confirms previous results (Stefan et al., 2000; Quartarone et al., 2003; Sale et al., 2007). The CSP is believed to have both a spinal and a cortical $\left(\mathrm{GABA}_{\mathrm{B}}\right.$ receptormediated) component (Werhahn et al., 1999). Spinal mechanisms contribute to EMG suppression in the first part of the silent period (<100 ms), as H-reflexes are suppressed (Fuhr et al., 1991; Uncini et al., 1993). The later part of the CSP, when motoneuron excitability is at pre-stimulus levels, is attributed to cortical inhibitory mechanisms (Inghilleri et al., 1993; Chen et al., 1999). The increase in CSP duration after PAS and with high cortisol levels (Figs. 3, 5) is therefore most likely the result of changes in cortical inhibition. CSP duration was increased after PAS, regardless of whether the MEP was facilitated. This is not surprising because the inhibitory processes responsible for the CSP are relatively independent of the extent of activation of corticospinal descending projection to motoneurons by TMS (Ho et al., 1998), and the CSP was assessed during voluntary activation, whereas PAS-induced MEP facilitation was assessed at rest.

SICI is believed to reflect $\mathrm{GABA}_{\mathrm{A}}$ inhibition (Ilić et al., 2002; Ziemann, 2003). There was no change in SICI after PAS, which supports previous findings (Ridding and Taylor, 2001; Stefan et al., 2002; Quartarone et al., 2003; Kujirai et al., 2006). Kujirai et al. (2006) found reduced SICI after PAS using a coil orientation that preferentially activated late I-waves, which are more susceptible to intracortical inhibition (Nakamura et al., 1997; Di Lazzaro et al., 1998; Hanajima et al., 1998). Therefore, it is possible that PAS may produce subtle changes in SICI not detected with the coil orientation we used.

SICI was not influenced by time of day (or cortisol levels) in experiment 1 and does not appear to modulate PAS effectiveness. Circadian variation in GABA release has been demonstrated in the mammalian brain (Castañeda et al., 2004; Gompf and Allen, 2004), and $\mathrm{GABA}_{\mathrm{A}}$-induced chloride currents are enhanced by metabolites of steroid hormones (Majewska et al., 1986). Our assessment of SICI may not be sufficiently sensitive to detect small time-of-day changes in SICI (see above). Alternatively, perhaps there is little modulation of GABA release (after PAS or at different times of day) onto $\mathrm{GABA}_{\mathrm{A}}$ receptors in the $\mathrm{M} 1$ circuitry acted on by paired-pulse TMS.

In conclusion, neuroplasticity induction in human M1 with PAS was more effective in the evening, when endogenous cortisol levels were low. Hydrocortisone administration in the evening raised circulating cortisol levels and impaired M1 neuroplasticity. PAS-induced LTP-like plasticity engages neural circuits involved in motor learning in humans (Ziemann et al., 2004; Stefan et al., 2006). Our findings have important implications for rehabilitative therapies using neuroplastic change in human M1 to promote functional recovery (Uy et al., 2003; Kim et al., 2006; McDonnell et al., 2007b), because they suggest that plasticity induction and, presumably, any therapeutic benefit would be enhanced when circulating cortisol levels are low.

\section{References}

Berardelli A, Inghilleri M, Rothwell JC, Romeo S, Currà A, Gilio F, Modugno N, Manfredi M (1998) Facilitation of muscle evoked responses after repetitive cortical stimulation in man. Exp Brain Res 122:79-84.

Butefisch CM, Davis BC, Wise SP, Sawaki L, Kopylev L, Classen J, Cohen LG (2000) Mechanisms of use-dependent plasticity in the human motor cortex. Proc Natl Acad Sci U S A 97:3661-3665.

Carroll TJ, Riek S, Carson RG (2001) Reliability of the input-output properties of the cortico-spinal pathway obtained from transcranial magnetic and electrical stimulation. J Neurosci Methods 112:193-202.

Castañeda TR, de Prado BM, Prieto D, Mora F (2004) Circadian rhythms of dopamine, glutamate and GABA in the striatum and nucleus accumbens of the awake rat: modulation by light. J Pineal Res 36:177-185.

Charlton CS, Ridding MC, Thompson PD, Miles TS (2003) Prolonged peripheral nerve stimulation induces persistent changes in excitability of human motor cortex. J Neurol Sci 208:79-85.

Chaudhury D, Wang LM, Colwell CS (2005) Circadian regulation of hippocampal long-term potentiation. J Biol Rhythms 20:225-236.

Chen R, Lozano AM, Ashby P (1999) Mechanism of the silent period following transcranial magnetic stimulation. Evidence from epidural recordings. Exp Brain Res 128:539-542.

Collins DR, Davies SN (1997) Melatonin blocks the induction of long-term potentiation in an N-methyl-D-aspartate independent manner. Brain Res 767:162-165.

Cotman CW, Engesser-Cesar C (2002) Exercise enhances and protects brain function. Exerc Sport Sci Rev 30:75-79.

Day BL, Thompson PD, Dick JP, Nakashima K, Marsden CD (1987) Different sites of action of electrical and magnetic stimulation of the human brain. Neurosci Lett 75:101-106.

de Quervain DJ, Roozendaal B, Nitsch RM, McGaugh JL, Hock C (2000) Acute cortisone administration impairs retrieval of long-term declarative memory in humans. Nat Neurosci 3:313-314.

Di Lazzaro V, Restuccia D, Oliviero A, Profice P, Ferrara L, Insola A, Mazzone P, Tonali P, Rothwell JC (1998) Magnetic transcranial stimulation at intensities below active motor threshold activates intracortical inhibitory circuits. Exp Brain Res 119:265-268.

Dubrovsky B, Williams D, Kraulis I (1985) Effects of corticosterone and 5 alpha-dihydrocorticosterone on brain excitability in the rat. J Neurosci Res 14:117-128.

Edwards S, Clow A, Evans P, Hucklebridge F (2001) Exploration of the awakening cortisol response in relation to diurnal cortisol secretory activity. Life Sci 68:2093-2103.

El-Sherif Y, Tesoriero J, Hogan MV, Wieraszko A (2003) Melatonin regulates neuronal plasticity in the hippocampus. J Neurosci Res 72:454-460.

Fratello F, Veniero D, Curcio G, Ferrara M, Marzano C, Moroni F, Pellicciari MC, Bertini M, Rossini PM, De Gennaro L (2006) Modulation of corticospinal excitability by paired associative stimulation: reproducibility of effects and intraindividual reliability. Clin Neurophysiol 117:2667-2674.

Fuhr P, Agostino R, Hallett M (1991) Spinal motor neuron excitability during the silent period after cortical stimulation. Electroencephalogr Clin Neurophysiol 81:257-262.

Gompf HS, Allen CN (2004) GABAergic synapses of the suprachiasmatic nucleus exhibit a diurnal rhythm of short-term synaptic plasticity. Eur J Neurosci 19:2791-2798.

Hanajima R, Ugawa Y, Terao Y, Sakai K, Furubayashi T, Machii K, Kanazawa 
I (1998) Paired-pulse magnetic stimulation of the human motor cortex: differences among I waves. J Physiol 509:607-618.

Hess G, Donoghue JP (1994) Long-term potentiation of horizontal connections provides a mechanism to reorganize cortical motor maps. J Neurophysiol 71:2543-2547.

Ho KH, Nithi K, Mills KR (1998) Covariation between human intrinsic hand muscles of the silent periods and compound muscle action potentials evoked by magnetic brain stimulation: evidence for common inhibitory connections. Exp Brain Res 122:433-440.

Hsu FC, Garside MJ, Massey AE, McAllister-Williams RH (2003) Effects of a single dose of cortisol on the neural correlates of episodic memory and error processing in healthy volunteers. Psychopharmacology 167:431-442.

Ilić TV, Meintzschel F, Cleff U, Ruge D, Kessler KR, Ziemann U (2002) Short-interval paired-pulse inhibition and facilitation of human motor cortex: the dimension of stimulus intensity. J Physiol 545:153-167.

Inghilleri M, Berardelli A, Cruccu G, Manfredi M (1993) Silent period evoked by transcranial stimulation of the human cortex and cervicomedullary junction. J Physiol 466:521-534.

Keel JC, Smith MJ, Wassermann EM (2001) A safety screening questionnaire for transcranial magnetic stimulation. Clin Neurophysiol 112:720.

Kim YH, You SH, Ko MH, Park JW, Lee KH, Jang SH, Yoo WK, Hallett M (2006) Repetitive transcranial magnetic stimulation-induced corticomotor excitability and associated motor skill acquisition in chronic stroke. Stroke 37:1471-1476.

Kujirai K, Kujirai T, Sinkjaer T, Rothwell JC (2006) Associative plasticity in human motor cortex during voluntary muscle contraction. J Neurophysiol 96:1337-1346.

Kujirai T, Caramia MD, Rothwell JC, Day BL, Thompson PD, Ferbert A, Wroe S, Asselman P, Marsden CD (1993) Corticocortical inhibition in human motor cortex. J Physiol 471:501-519.

Kusuki T, Imahori Y, Ueda S, Inokuchi K (1997) Dopaminergic modulation of LTP induction in the dentate gyrus of intact brain. Neuroreport 8:2037-2040.

Majewska MD, Harrison NL, Schwartz RD, Barker JL, Paul SM (1986) Steroid hormone metabolites are barbiturate-like modulators of the GABA receptor. Science 232:1004-1007.

Mariorenzi R, Zarola F, Caramia MD, Paradiso C, Rossini PM (1991) Noninvasive evaluation of central motor tract excitability changes following peripheral nerve stimulation in healthy humans. Electroencephalogr Clin Neurophysiol 81:90-101.

McDonnell MN, Orekhov Y, Ziemann U (2007a) Suppression of LTP-like plasticity in human motor cortex by the GABA(B) receptor agonist baclofen. Exp Brain Res 180:181-186.

McDonnell MN, Hillier SL, Miles TS, Thompson PD, Ridding MC (2007b) Influence of combined afferent stimulation and task-specific training following stroke: a pilot randomized controlled trial. Neurorehabil Neural Repair 21:435-443.

Meunier S, Russmann H, Simonetta-Moreau M, Hallett M (2007) Changes in spinal excitability after PAS. J Neurophysiol 97:3131-3135.

Morgante F, Espay AJ, Gunraj C, Lang AE, Chen R (2006) Motor cortex plasticity in Parkinson's disease and levodopa-induced dyskinesias. Brain 129:1059-1069.

Müller JF, Orekhov Y, Liu Y, Ziemann U (2007) Homeostatic plasticity in human motor cortex demonstrated by two consecutive sessions of paired associative stimulation. Eur J Neurosci 25:3461-3468.

Murakami T, Sakuma K, Nomura T, Uemura Y, Hashimoto I, Nakashima K (2008) Changes in somatosensory-evoked potentials and high-frequency oscillations after paired-associative stimulation. Exp Brain Res 184:339-347.

Nakamura H, Kitagawa H, Kawaguchi Y, Tsuji H (1997) Intracortical facilitation and inhibition after transcranial magnetic stimulation in conscious humans. J Physiol 498:817-823.

Newcomer JW, Selke G, Melson AK, Hershey T, Craft S, Richards K, Alderson AL (1999) Decreased memory performance in healthy humans induced by stress-level cortisol treatment. Arch Gen Psychiatry 56:527-533.

Pascual-Leone A, Valls-Solé J, Wassermann EM, Hallett M (1994) Responses to rapid-rate transcranial magnetic stimulation of the human motor cortex. Brain 117:847-858.

Quartarone A, Bagnato S, Rizzo V, Siebner HR, Dattola V, Scalfari A, Mor- gante F, Battaglia F, Romano M, Girlanda P (2003) Abnormal associative plasticity of the human motor cortex in writer's cramp. Brain 126:2586-2596.

Raghavan AV, Horowitz JM, Fuller CA (1999) Diurnal modulation of longterm potentiation in the hamster hippocampal slice. Brain Res 833:311-314.

Ranjit N, Young EA, Raghunathan TE, Kaplan GA (2005) Modeling cortisol rhythms in a population-based study. Psychoneuroendocrinology 30:615-624.

Ridding MC, Flavel SC (2006) Induction of plasticity in the dominant and non-dominant motor cortices of humans. Exp Brain Res 171:551-557.

Ridding MC, Taylor JL (2001) Mechanisms of motor-evoked potential facilitation following prolonged dual peripheral and central stimulation in humans. J Physiol 537:623-631.

Ridding MC, McKay DR, Thompson PD, Miles TS (2001) Changes in corticomotor representations induced by prolonged peripheral nerve stimulation in humans. Clin Neurophysiol 112:1461-1469.

Rioult-Pedotti MS, Friedman D, Donoghue JP (2000) Learning-induced LTP in neocortex. Science 290:533-536.

Sale MV, Ridding MC, Nordstrom MA (2007) Factors influencing the magnitude and reproducibility of corticomotor excitability changes induced by paired associative stimulation. Exp Brain Res 181:615-626.

Smith MJ, Keel JC, Greenberg BD, Adams LF, Schmidt PJ, Rubinow DA, Wassermann EM (1999) Menstrual cycle effects on cortical excitability. Neurology 53:2069-2072.

Starkman MN, Gebarski SS, Berent S, Schteingart DE (1992) Hippocampal formation volume, memory dysfunction, and cortisol levels in patients with Cushing's syndrome. Biol Psychiatry 32:756-765.

Stefan K, Kunesch E, Cohen LG, Benecke R, Classen J (2000) Induction of plasticity in the human motor cortex by paired associative stimulation. Brain 123:572-584.

Stefan K, Kunesch E, Benecke R, Cohen LG, Classen J (2002) Mechanisms of enhancement of human motor cortex excitability induced by interventional paired associative stimulation. J Physiol 543:699-708.

Stefan K, Wycislo M, Classen J (2004) Modulation of associative human motor cortical plasticity by attention. J Neurophysiol 92:66-72.

Stefan K, Wycislo M, Gentner R, Schramm A, Naumann M, Reiners K, Classen J (2006) Temporary occlusion of associative motor cortical plasticity by prior dynamic motor training. Cereb Cortex 16:376-385.

Tecchio F, Zappasodi F, Pasqualetti P, De Gennaro L, Pellicciari MC, Ercolani M, Squitti R, Rossini PM (2008) Age dependence of primary motor cortex plasticity induced by paired associative stimulation. Clin Neurophysiol 119:675-682.

Tononi G, Cirelli C (2006) Sleep function and synaptic homeostasis. Sleep Med Rev 10:49-62.

Uncini A, Treviso M, Di Muzio A, Simone P, Pullman S (1993) Physiological basis of voluntary activity inhibition induced by transcranial cortical stimulation. Electroencephalogr Clin Neurophysiol 89:211-220.

Uy J, Ridding MC, Hillier S, Thompson PD, Miles TS (2003) Does induction of plastic change in motor cortex improve leg function after stroke? Neurology 61:982-984.

Vyazovskiy VV, Cirelli C, Pfister-Genskow M, Faraguna U, Tononi G (2008) Molecular and electrophysiological evidence for net synaptic potentiation in wake and depression in sleep. Nat Neurosci 11:200-208.

Werhahn KJ, Kunesch E, Noachtar S, Benecke R, Classen J (1999) Differential effects on motorcortical inhibition induced by blockade of GABA uptake in humans. J Physiol 517:591-597.

Whitlock JR, Heynen AJ, Shuler MG, Bear MF (2006) Learning induces long-term potentiation in the hippocampus. Science 313:1093-1097.

Wolters A, Sandbrink F, Schlottmann A, Kunesch E, Stefan K, Cohen LG, Benecke R, Classen J (2003) A temporally asymmetric Hebbian rule governing plasticity in the human motor cortex. J Neurophysiol 89:2339-2345.

Ziemann U (2003) Pharmacology of TMS. Suppl Clin Neurophysiol $56: 226-231$.

Ziemann U, Ilić TV, Pauli C, Meintzschel F, Ruge D (2004) Learning modifies subsequent induction of long-term potentiation-like and long-term depression-like plasticity in human motor cortex. J Neurosci 24:16661672. 